\title{
A prevalência de doenças crônicas não transmissíveis em usuários de um centro de atenção psicossocial II
}

\author{
The prevalence of noncommunicable chronic diseases in users of a psychosocial care center II \\ La prevalencia de enfermedades crónicas no transmisibles em usuarios de un centro de atención \\ psicosocial II
}

Recebido: 01/06/2021 | Revisado: 07/06/2021 | Aceito: 09/06/2021 | Publicado: 23/06/2021

\author{
Pablo Alexandre Silva \\ ORCID: https://orcid.org/0000-0002-4350-4829 \\ Universidade Federal do Rio de Janeiro, Brasil \\ E-mail: pabloales81@gmail.com \\ Silvia Regina Carvalho de Souza da Silva \\ ORCID: https://orcid.org/0000-0003-2497-9930 \\ Universidade do Estado do Rio de Janeiro, Brasil \\ E-mail: silvia.silva@ souunisuam.com.br \\ Beatriz Neves Borges \\ ORCID: https://orcid.org/0000-0002-3217-4545 \\ Universidade Federal do Rio de Janeiro, Brasil. \\ E-mail: bianevesborges@gmail.com \\ Jéssica do Nascimento Rezende \\ ORCID: https://orcid.org/0000-0002-0018-161X \\ Universidade Federal Fluminense, Brasil \\ E-mail: jessiiica_rezende@ @otmail.com \\ Leila Vianna dos Reis \\ ORCID: https://orcid.org/0000-0001-6173-8832 \\ Universidade Federal do Rio de Janeiro, Brasil \\ E-mail: lviannareis@gmail.com \\ Lucimara Lopes Rase \\ ORCID: https://orcid.org/0000-0002-9502-0041 \\ Universidade do Estado do Rio de Janeiro, Brasil \\ E-mail: lucimara.rase@gmail.com
}

\begin{abstract}
Resumo
Os CAPS II são Centros de Atenção Psicossocial tem como objetivo reinserir o sujeito na sociedade, promover autonomia e um cuidado de maneira integral ao sujeito. Deste modo, observamos que muitos usuários inseridos nestes serviços são portadores de Doenças Crônicas não Transmissíveis (DCNTs), que de modo geral são relacionadas a causas múltiplas e diversos fatores de risco que influenciam no cotidiano, do ponto de vista econômico à qualidade de vida destes indivíduos. Os objetivos do presente estudo são: traçar o perfil dos usuários atendidos no CAPS; verificar a prevalência de Doenças Crônicas Não Transmissíveis com enfoque na Hipertensão Arterial Sistêmica (HAS) e no Diabetes Mellitus (DM), na amostra apresentada; identificar os fatores de risco presentes na amostra estudada para desenvolvimento das DCNTs. Trata-se, portanto, de um estudo documental, exploratório descritivo e retrospectivo com análise quantitativa. A coleta se deu de modo retrospectivo pelas fichas de acolhimento de uma unidade de CAPS II, localizado na cidade do Rio de Janeiro, no período de março a agosto de 2020. A amostra foi composta por 81 usuários, onde destes apenas 29 tinham registros sobre DCNTs em suas fichas, com taxa de prevalência de 35,8\%, a maior taxa se deu em indivíduos do sexo feminino, $40 \%$. Conclui-se a necessidade de dinamização das informações acerca do estado clínico de cada usuário a fim de fornecer uma assistência integral, voltada à promoção de saúde e continuidade no cuidado de pessoas com transtornos mentais.
\end{abstract}

Palavras-chave: Saúde mental; Doenças crônicas não transmissíveis; Centros de atenção psicossocial; Clínica de família.

\begin{abstract}
PCC II are Psychosocial Care Centers with the objective of reinserting the subject in society, promoting autonomy and a comprehensive care for the subject. Thus, we observe that many users inserted in these services have Chronic NonCommunicable Diseases (NCDs), which are generally related to multiple causes and various risk factors that influence their daily lives, from an economic point of view, to their quality of life. individuals. The objectives of the present study are: to outline the profile of users assisted by the CAPS; to verify the prevalence of Chronic Non-Communicable Diseases with a focus on Systemic Arterial Hypertension (SAH) and Diabetes Mellitus (DM), in the presented sample;
\end{abstract}


identify the risk factors present in the studied sample for the development of CNCDs. It is, therefore, a documentary, exploratory, descriptive and retrospective study with quantitative analysis. The collection took place retrospectively through the reception forms of a CAPS II unit, located in the city of Rio de Janeiro, from March to August 2020. The sample consisted of 81 users, of which only 29 had records about NCDs in their records, with a prevalence rate of $35.8 \%$, the highest rate occurred in females, $40 \%$. It concludes the need to streamline information about the clinical status of each user in order to provide comprehensive care, aimed at promoting health and continuity in the care of people with mental disorders.

Keywords: Mental health; Noncommunicable chronic diseases; Psychosocial care centers; Family clinic.

\section{Resumen}

Los CAPS II son Centros de Atención Psicosocial con el objetivo de reinsertar al sujeto en la sociedad, promoviendo la autonomía y la atención integral del sujeto. Así, observamos que muchos usuarios insertados en estos servicios tienen Enfermedades Crónicas No Transmisibles (ECNT), las cuales generalmente se relacionan con múltiples causas y diversos factores de riesgo que influyen en su día a día, desde el punto de vista económico, a su calidad de vida. .individuos. Los objetivos del presente estudio son: perfilar el perfil de los usuarios atendidos en CAPS; verificar la prevalencia de Enfermedades Crónicas No Transmisibles con enfoque en Hipertensión Arterial Sistémica (HSA) y Diabetes Mellitus (DM), en la muestra presentada; identificar los factores de riesgo presentes en la muestra estudiada para el desarrollo de ENT. Se trata, por tanto, de un estudio documental, exploratorio, descriptivo y retrospectivo con análisis cuantitativo. La recolección se realizó de manera retrospectiva a través de los formularios de recepción de una unidad CAPS II, ubicada en la ciudad de Río de Janeiro, de marzo a agosto de 2020. La muestra estuvo conformada por 81 usuarios, de los cuales solo 29 tenían registros sobre ECNT en sus registros, con una tasa de prevalencia del 35,8\%, la tasa más alta ocurrió en mujeres, $40 \%$. Se concluye la necesidad de agilizar la información sobre el estado clínico de cada usuario con el fin de brindar una atención integral, orientada a promover la salud y la continuidad en la atención de las personas con trastornos mentales.

Palabras clave: Salud mental; Enfermedades crónicas no transmisibles; Centros de atención psicosocial; Clínica familiar.

\section{Introdução}

Doenças crônicas de modo geral estão relacionadas a causas múltiplas. Aquelas dadas como não transmissíveis englobam um conjunto de condições crônicas. Apresentam curso gradual e tem longo ou indefinido período, podendo apresentar variações, possíveis períodos de agudização, o que pode gerar incapacidades. Nem sempre apresentam cura, seu processo de cuidado pode ser de curso lento e deve ser contínuo para que se obtenha melhor qualidade de vida, considerando as opções de intervenção que gerem cuidados à saúde do indivíduo afetado (Brasil, 2013).

As Doenças crônicas não transmissíveis (DCNTs), são responsáveis por cerca de 70\% dos óbitos registrados em todo mundo. Tais doenças apresentam grande impacto econômico e no convívio familiar, social e individual, estas afetam em maior frequência a população de baixa renda que no Brasil está mais exposta aos fatores de risco e acessa menos os serviços de saúde, o que reflete negativamente na qualidade de vida do sujeito (Malta, et al., 2020).

De acordo com estes fatos sabemos que o controle as DCNTs é um desafio global. Consideramos os grupos de grande magnitude que englobam: o câncer, o diabetes, as doenças circulatórias e respiratórias. Entre os principais fatores de risco considerados estão: o tabagismo, uso abusivo de álcool, inatividade física e alimentação não saudável

(Andreis et al, 2019). Ao verificarmos tais fatores de risco, observamos no dia a dia dos serviços de saúde como os Centros de Atenção Psicossocial II (CAPS II), que muitos destes estão presentes na vida dos usuários, agravando ou gerando consequências a saúde destes indivíduos.

Levando em consideração a contextualização exposta, verifica-se a relevância dos CAPS II, que são Centros de Atenção Psicossocial, oriundos do movimento da reforma psiquiátrica e que tem, dentre outros pilares que os fundam, o objetivo de reinserir o sujeito na sociedade, promover autonomia e a integralidade dos cuidados, de modo a trabalhar para a sua reabilitação psicossocial. Neste contexto, o público inscrito nestas unidades é tomado como principal foco da incidência das ações, seja quando o tomamos como protagonista social seja na corresponsabilização pela promoção de seu autocuidado. Contudo, 
identificamos que o exercício destas prerrogativas ainda esbarram com algumas dificuldades cotidianas que afetam o cuidado na saúde (Nasi \& Schneider, 2011).

Sabemos que o sujeito inserido no serviço de saúde mental não está limitado ao seu diagnóstico clínico; ele também é afetado pelos mais diversos fatores e, dentre eles, estão os sociais, psicológicos, biológicos, econômicos e ambientais. Desta feita, convém que as diretrizes em saúde não se estabeleçam de forma rígida, tomando o sujeito de forma totalitária erroneamente (Secretária de estado de saúde do Espírito Santo, 2018).

Destacamos que o usuário do serviço de saúde mental comumente apresenta dificuldades em relação ao autocuidado, o que contribui de certa forma para o surgimento e agravamento de comorbidades. O registro das informações relacionadas a condições clínicas é muito escasso e, considerando especialmente os usuários dos serviços de saúde mental, tais dados são significativos, pois estes usuários apresentam grande vulnerabilidade. Neste sentido, é de suma importância a promoção da atenção integral à saúde (Branquinho et al, 2014).

Auxilia-nos a pensar o usuário dos serviços de saúde mental como um sujeito dinâmico e com diversas necessidades, de modo a visualizá-lo como um todo, identificando e atendendo suas necessidades, fazendo com que os profissionais articulados à Rede de Atenção Psicossocial (RAPS) possam intervir de modo conjunto e articulado, visando a melhoria de suas condições de estar em sociedade e de sua qualidade de vida. Conhecer os fatores de risco que envolvem os usuários deste serviço, verificar a prevalência das DCNTs que estes apresentam, são fatores que facilitam na criação de estratégias que direcionem o cuidado, a promoção da saúde e a prevenção de riscos e aquisição de novas comorbidades desta população. Tais medidas facilitam o dia a dia do profissional que atua diretamente com estes usuários, aprimorando a comunicação e o cuidado, de forma abrangente e conforme as demandas apresentadas pelo sujeito. (Chiavagatti, et al., 2012) (Próchno \& Bessa, 2016).

Identificar os fatores de risco e realizar vigilância em DCNTs dão a dimensão e nos permite avaliar o quão arriscado é para a população de modo em geral e, sobretudo, o perfil comportamental diante da amostra estudada, o que fornece subsídios para criação das estratégias de promoção à saúde e prevenção, além de facilitar a abordagem ao sujeito e a intensificação de seus cuidados (Brasil, 2005).

Com base no exposto, os objetivos do presente estudo podem ser contemplados com as seguintes proposições: Traçar o perfil dos usuários atendidos no CAPS; verificar a prevalência de Doenças Crônicas Não Transmissíveis com enfoque na Hipertensão Arterial Sistêmica (HAS) e no Diabetes Mellitus (DM), na amostra apresentada; identificar os fatores de risco presentes na amostra estudada para desenvolvimento das DCNTs.

\section{Metodologia}

Este estudo buscou realizar o levantamento de dados acerca do tema proposto - doenças crônicas não transmissíveis e sua relação com usuários do serviço de saúde mental - com o objetivo de traçar resultados e obter esclarecimentos, em busca de conhecimentos que apresentem soluções ou novas visões. Sendo este estudo de cunho documental, exploratório-descritivo e retrospectivo com análise quantitativa, objetivamos informar sobre a distribuição de um evento na amostra em termos quantitativos. O que se justifica pela apresentação em números, exemplificados por gráficos que são complementados qualitativamente com informações que facilitam o entendimento, afim de que se compreenda de forma efetiva o que se apresenta na amostra deste estudo (Pereira, et al., 2018).

Foi analisada uma amostra representada por usuários do acolhimento de primeira vez do cenário da pesquisa, CAPS II Rubens Corrêa, pelo período de 6 meses retrospectivos, referentes ao ano de 2020. De forma mais precisa, os meses de março a agosto. Com parecer positivo do Comitê de Ética em Pesquisa (CEP) do Instituto de Psiquiatria da Universidade Federal do Rio de Janeiro sob o número CAEE: 40126720.0.0000.5263 e do CEP da Secretária Municipal de Saúde do Rio de Janeiro sob o número CAAE: 40126720.0.3001.5279. 
A coleta de dados ocorreu a partir do registro realizado pela equipe da unidade nas fichas de acolhimento e evoluções complementares utilizadas no serviço, não havendo contato de forma direta com os participantes dado o fato da pesquisa ser realizada por método retrospectivo. O que vai de acordo com a resolução do Conselho de Saúde 466/12, o que garante o anonimato dos participantes, após autorização da pesquisa pelos comitês de ética em pesquisa.

Foram utilizadas fichas de coletas de dados a fim de organizar as informações para que posteriormente se fizesse a tabulação por meio do programa Excel e análise dos mesmos. Os dados coletados respeitaram os critérios de inclusão para a amostra do estudo, a saber, os usuários em acolhimento de primeira vez nesta unidade de CAPS II e usuários com registro na ficha de acolhimento das clínicas da família de referência. Foram excluídos os usuários encaminhados às outras unidades de referência e usuários em retorno para acolhimento de segunda vez, valendo-nos da data de início do estudo.

Por fim, para resultados precisos, utilizou-se fórmulas estatísticas para avaliar as frequências simples e os percentuais encontrados, bem como a formulação para o recolhimento da taxa de prevalência que considera os usuários que respeitaram os critérios anteriores sobre o número de usuários que apresentaram DCNTs registradas em suas fichas de acolhimento.

\section{Resultados}

No período proposto para o estudo, o CAPS II recebeu 81 acolhimentos de primeira vez. Utilizando dos critérios de inclusão e exclusão, todo o quantitativo foi considerado para análise. Destes, a média de idade encontrada foi de aproximadamente 41 anos (41,43), sendo a maior idade de 73 anos e a menor idade encontrada de 19 anos. Quando se questiona o registro de dados como cor e raça, nenhumas das fichas de acolhimento obteve esse dado em seu registro, ou seja, 100\% dos casos não tinham essa informação.

Sabendo que, dentro da Rede de Atenção Psicossocial as Clínicas da família, os centros municipais de saúde têm papel fundamental no acompanhamento de usuários com doenças crônicas não transmissíveis e, principalmente, dão suporte aos CAPS para que se compreenda o sujeito de forma integral, busca-se o registro das clínicas ou CMS's que dão suporte a este serviço a fim de obtermos informações sobre o acompanhamento dos usuários pelos serviços da rede. Sendo assim, 79 do total de fichas analisadas tinham a clínica da família ou CMS de referência, apenas 2 não obtinham essa informação e destes que possuíam o dado, apenas 9 apresentavam a situação de acompanhamento regular, todo o restante encontrava-se, portanto, sem informações.

Apresentamos na sequência os resultados exemplificados em gráficos, que demonstram as razões de prevalência de acordo com o encontrado na coleta de dados. 
Gráfico 1: Distribuição da taxa de prevalência de acordo com o sexo dos usuários de acolhimento de primeira vez do Centro de atenção psicossocial II Rubens Corrêa. Rio de Janeiro, RJ, 2021.

\section{Taxa de prevalência de acordo com o Sexo}

60

50

40

30

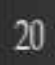

10

20

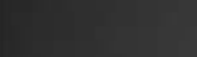

0
55

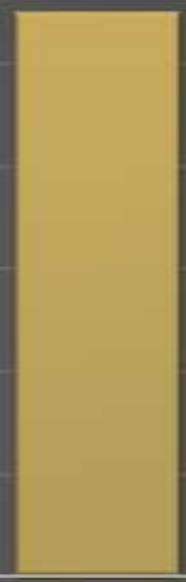

40

Masculino

Feminino

r Quantidade Total

IUsuários com registro de doenças crônicas não transmissiveis

r Taxa de Prevalência

Fonte: Autores (2021).

O gráfico acima apresenta a relação entre os usuários de acolhimento de primeira vez, que apresentaram doenças crônicas não transmissíveis independente da etiologia, identificando o sexo por eles indicado e articulando a taxa de prevalência de cada um.

Destaco que o quantitativo de usuários do sexo feminino foi superior aos do sexo masculino, o que define uma taxa de prevalência superior para esta categoria. Foram encontrados, dentre as 81 fichas de acolhimento, 26 do sexo masculino (M) e 55 do sexo feminino (F). Sendo que, dentre estes, 22 do sexo F e 7 do sexo M apresentaram na ficha dados sobre DCNTs, resultando em uma taxa de prevalência de $40 \%$ (F) e 26,92 (M).

Saliento que mulheres apresentam mais riscos de desenvolvimento de doenças "silenciosas" como a hipertensão e que as mesmas apresentam maiores fatores de risco. Quando se trata de saúde mental, estas mulheres são muitas vezes silenciadas em seus corpos, o que dificulta um diagnóstico precoce ou intervenção para promoção de saúde. 
Gráfico 2: Registro de Doenças Crônicas não Transmissíveis dos usuários de acolhimento de primeira vez do Centro de atenção psicossocial II Rubens Corrêa. Rio de Janeiro, RJ, 2021.

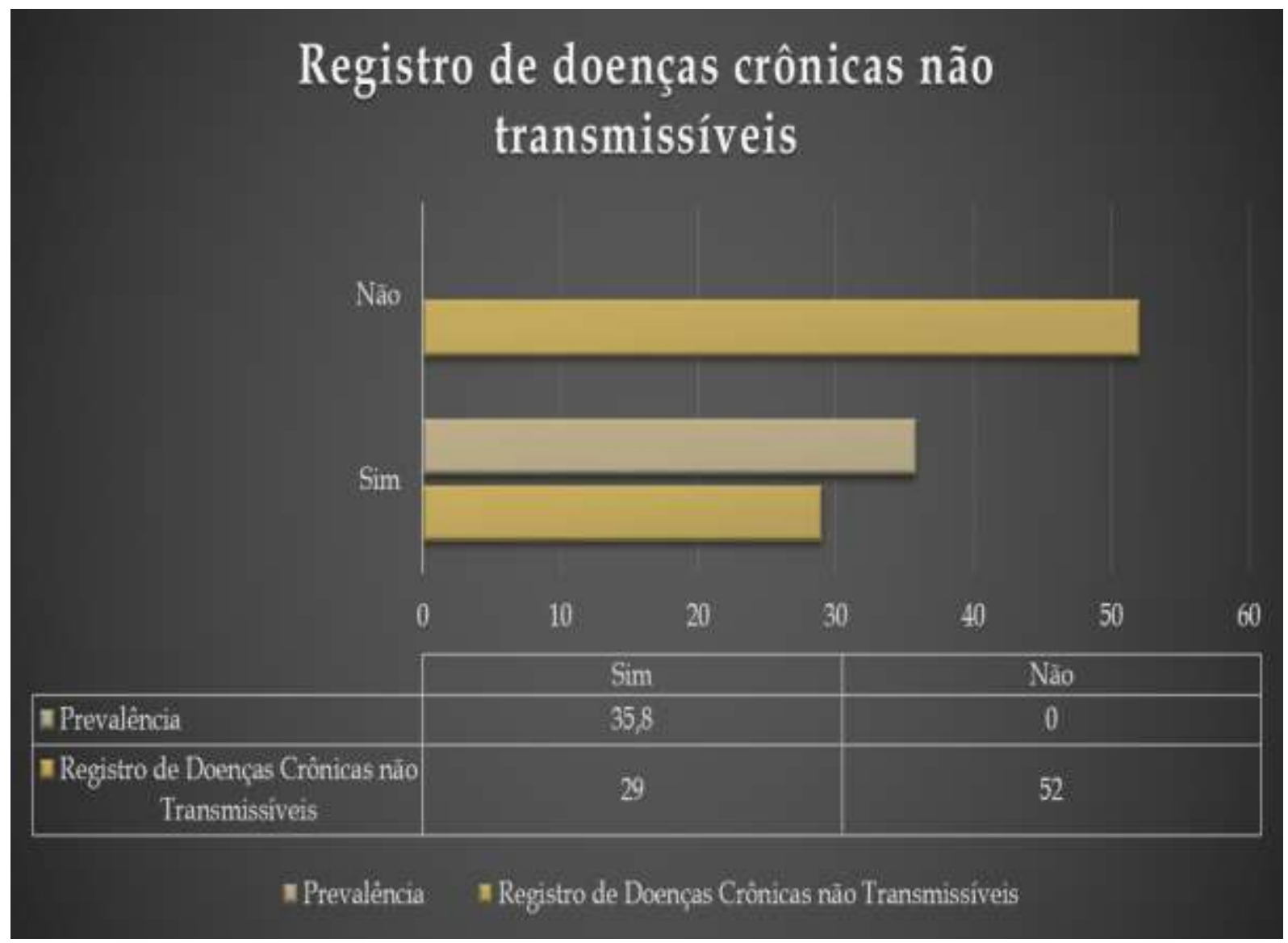

Fonte: Autores (2021).

Este gráfico representa o quantitativo de registros de doenças crônicas não transmissíveis nas fichas de acolhimento de primeira vez, sendo que o maior valor apresentado foi de 52, onde não haviam registros, seguido pelos efetivos registros que representam 29 destes, que apresentavam registros e a taxa de prevalência de 35,8\%.

Tal taxa representa $1 / 3$ do valor total encontrado, o que é de certa forma um índice pequeno quando analisado de forma global. Esse percentual é de suma importância para que se identifique o ponto de partida das faltas de registro, possibilitando um questionamento a partir da taxa apresentada que, considerando o grau de adoecimento da sociedade, é extremamente grave, pois podemos identificar casos subnotificados nas mais diversas áreas, além de dificultar o tratamento e acompanhamento de forma integral destes sujeitos. 
Gráfico 3: Registro do uso de medicações dos usuários de acolhimento de primeira vez do Centro de atenção psicossocial II Rubens Corrêa. Rio de Janeiro, RJ, 2021.

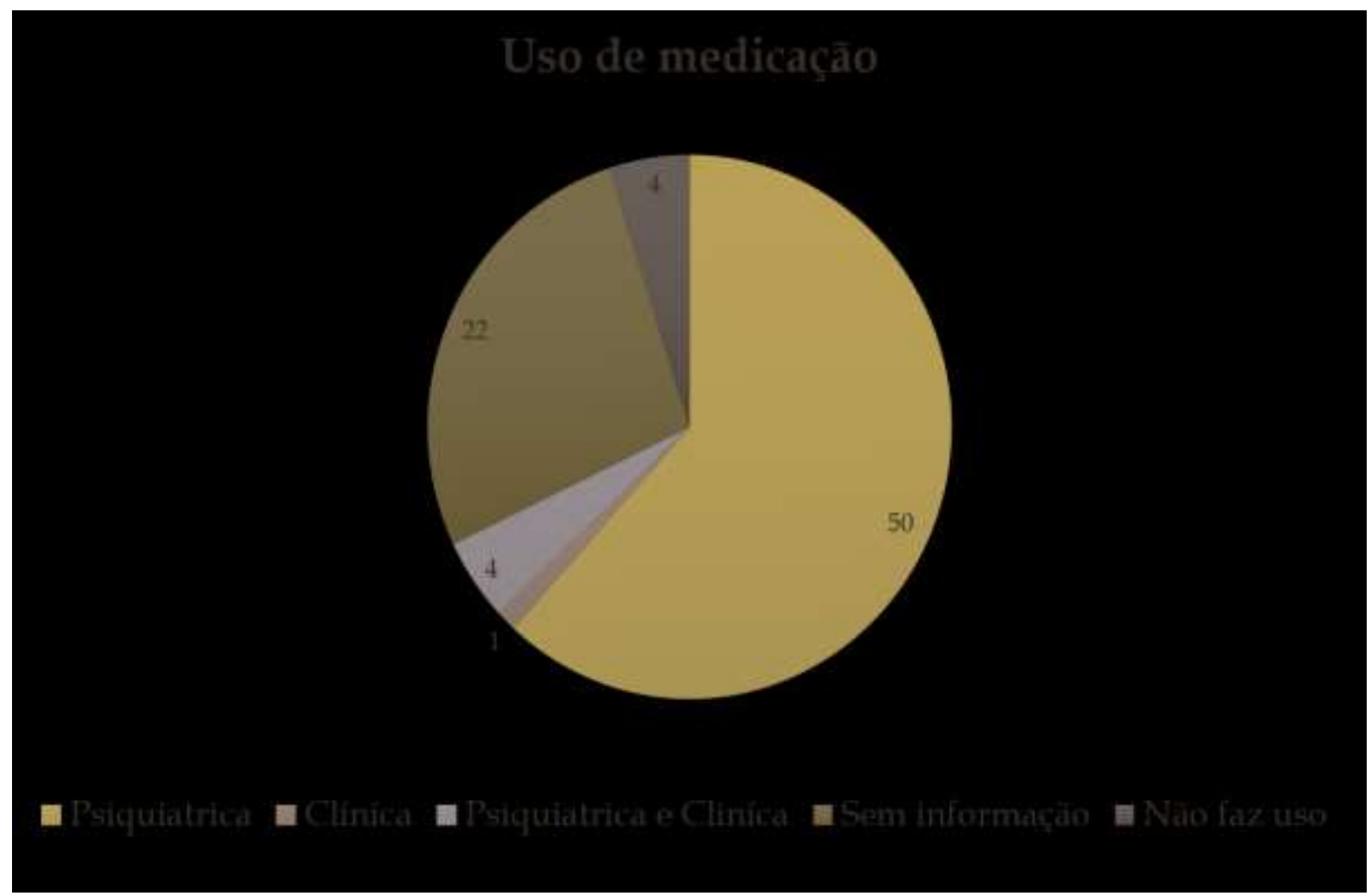

Fonte: Autores (2021).

Nas fichas de acolhimento pudemos avaliar as medicações utilizadas. Por vezes o usuário nega apresentar alguma comorbidade, mas, ao informar os medicamentos, podemos identificar que fazem uso de medicações clínicas além das prescritas por psiquiatras, sendo possível a verificação dos valores que serão apresentados a seguir. Usuários que possuem comorbidades diagnosticadas, por vezes não fazem a utilização de medicamentos de forma regular, o que causa danos severos à saúde física, além de prejudicar a saúde mental do indivíduo.

Consideramos, então, o importante destaque ao pequeno valor representado por apenas 1 usuário que faz uso de medicação clínica e 4 usuários que fazem uso combinado de medicações clínicas e psiquiátricas, dentro da taxa de prevalência que aqui se representou, questionamos o fato de que esta é maior e, quando comparada a taxa de prevalência do uso de medicações que se representa pelo valor de 17,24\%, a mesma é aproximadamente 1/6 do representativo.

Dentre os usuários que apresentaram em suas fichas o relato de doenças crônicas não transmissíveis, aparecerem em destaque 9 destes com hipertensão e 4 diabéticos. Porém, em análise, apenas 20 das fichas tiveram informações complementares como o tipo de doença diagnosticada. Sabendo que dentre estes resultados podemos identificar os diversos fatores de risco apresentados por uma população, considerando o quantitativo total de informações, apenas 4 fichas relataram o tabagismo e o uso abusivo de álcool e 77 delas não obtiveram tal informação. Estes são dados importantes para que se avalie as condições de vida dos usuários, além de poder pensar de forma individual o projeto de acompanhamento de cada sujeito.

\section{Discussão}

O presente estudo levantou dados da saúde e do acompanhamento acerca dos cuidados ao usuário dos serviços de saúde mental, contemplando as mais diversas áreas de atuação da enfermagem que se interligam visto que a formação se faz para um acompanhamento de modo integral e uma visão total de cada sujeito que é assistido dentro dos serviços. 
Dentre os resultados apresentados, constatamos que o dado cor e raça não foi considerado nos registros das fichas de acolhimento deste CAPS II. Ao analisarmos tal fato, percebemos a relação importante a ser considerada, já que pessoas negras em estudos que comparam a autorreferência de cor e raça e DCNTs apresentam maiores prevalências em relação as pessoas brancas, além de serem mais prejudicadas quando o assunto se trata dos serviços de saúde, sofrendo restrições para o acesso aos mesmos. Estudos apontam que pessoas negras apresentam maiores fatores de risco para desenvolvimento de DCNTs, em que pese a baixa produção sobre tal assunto (Malta, Moura \& Bernal, 2015).

Piores condições de saúde se apresentam na população negra, devendo-se a fatores socioeconômicos e culturais na maior parte dos casos (Malta, et al., 2017). Tais disparidades são encontradas nos serviços públicos de saúde onde a população negra - cujo enfoque este levantamento não se propõe a realizar - mas que é extremamente prejudicada e tem fatores quantitativos de risco importantes para desenvolvimentos de DCNTs e outras doenças, sobretudo quando se estratifica dados de cor e raça, principalmente nos CAPS (Silva, et al., 2017).

A prevalência no sexo feminino se deu de forma superior ao sexo masculino neste âmbito, sendo de $40 \%$ a taxa encontrada. Tal valor aqui representado nos direcionam ao fato de que estudos apresentam taxas maiores para internações, acompanhamentos e presença de fatores de risco ao desenvolvimento de DCNTs no sexo feminino (Santos, et al., 2013). Porém, tal taxa, quando analisada dentro deste estudo, necessita alguma interpretação cautelosa, uma vez que muitas das fichas encontravam-se sem registro de informações.

A taxa de prevalência encontrada foi de $35,8 \%$, importante destacar que essa taxa faz representação para apenas $1 / 3$ da população estudada, pois há alguma dificuldade quanto aos registros destas informações, podendo tal situação se dar pelo fato de usuários de saúde mental não possuírem informações clínicas, serem negligenciados, terem seus corpos silenciados quando se trata de um acompanhamento integral, quando se trata de olhar o usuário como um todo. Os usuários destes serviços são por vezes destituídos da posição de sujeitos na cultura e tal fato produz reflexos na clínica, especialmente enquanto não estabelecermos um conjunto de práticas que integralizem os serviços e articulem a rede os corpos seguirão sendo silenciados (Santos, 2009) (Campos, 2001).

Dados de prevalência para DCNTs são mais identificados em pessoas com baixa escolaridade por exemplo, segundo os estudos. São também mais presentes em usuários dos serviços públicos de saúde. Pensando neste contexto, podemos verificar que, dentro dos serviços de saúde mental, a população se apresenta com maiores índices de vulnerabilidade, o que geram mais fatores predisponentes para o desenvolvimento das DCNTs (Malta, et al., 2017).

A prevalência do uso de medicações entre os usuários com diagnósticos de DCNTs dentro deste estudo representa aproximadamente 13\%. Quando comparado, o uso de medicações tem prevalência menor que do número total de diagnosticados, chegando por vezes a depender da doença a $40 \%$ de uso contínuo. Consideramos fatos como a dificuldade de acesso aos serviços de saúde como elemento hipotético para que o tratamento seja prejudicado (Tavares, et al., 2015).

França et al, conceitua que as DCNTs não possuem uma relação direta com os transtornos mentais, porém identifica que fatores de risco como a alimentação inadequada e inatividade física principalmente, são características comuns nos serviços de saúde mental. O que predispõe o usuário deste serviço ao desenvolvimento de DCNTs ou agravamento das mesmas. Além disso, identifica que muitos dos pacientes que possuíam dados de acompanhamento dos níveis pressóricos elevados, não possuíam diagnóstico para HAS e usuários com DM faziam parte da maior taxa de prevalência entres as DCNTs consideradas (França, et al., 2021). Estes dados corroboram com nossos objetivos que visam identificar dentro destes as taxas mais prevalentes e além de corroborar com outros estudos que conceituam maiores prevalências de HAS e DM na população em geral.

De acordo com os dados apresentados, sabemos da necessidade de acompanhamento de forma integral dos usuários dos serviços de saúde mental. Pensar a integralidade permite acolher com uma vertente assistencial cada indivíduo para que assim se construam as RAPS. Vendo o sujeito como um agente principal do seu cuidado, inserido dentro do contexto social e familiar 
e buscando as mudanças em seus tratamentos, cabe neste sentido a orientação e a organização do acesso aos serviços da rede pela equipe que o acompanha. Esta perspectiva nos remete a conceituação da clínica ampliada, que pensa o cuidado visando o encontro do serviço com a comunidade de acordo com o que é apresentado pelo usuário (Nasi, et al., 2009).

Ao refletirmos sobre o conceito de integralidade e as possibilidades para acompanhamento das DCNTs, constatamos que, as CF's e/ou CMS estão inseridas normalmente mais próximas ao território de atuação, além de estarem mais próximas também aos CAPS, podendo e necessitando de atuação conjunta entre os serviços, através dos Núcleo de apoio da saúde da família (NASF) e o matriciamento (França, et al., 2021). Daí a importância vista nos resultados que apresentaram quase $100 \%$ das fichas com os dados das unidades de referência, salientando ainda que os dados de acompanhamento são escassos, o que implica a possibilidade de continuidade no apoio quando se apresentam os prontuários dos usuários após o acolhimento de primeira vez.

Salientamos a importância de alguma mudança na concepção do acolhimento integral ao usuário, no intuito de que alcemos a possibilidade de um cuidado efetivo aos sujeitos envolvidos Dar lugar, voz e possibilidade para que o sujeito seja visto em sua totalidade, não mais o silenciando em estruturas fechadas do exercício clínico, mas acolhendo todas as características que o compõem como raça/cor, gênero ou condições socioeconômicas, por exemplo, viabiliza aos serviços da RAPS um conjunto de ações que direcionem um cuidado ampliado ao usuário, lançando luz sobre o campo que lhe foi silenciado.

\section{Considerações Finais}

Este estudo nos possibilitou dinamizar e verificar o quantitativo de registros feitos em um primeiro contato com os usuários de saúde mental, podendo assim identificar, dentro dos limites que as pesquisas apresentam, que se faz necessário mais informações acerca da saúde clínica de pessoas com transtornos mentais. A taxa de prevalência aqui sinalizada é um representativo menor do que o esperado para as altas taxas de prevalência apresentadas em outros estudos. Este valor tem sua justificativa amparada na falta de informações vistas nas fichas quando estratificado durante a coleta de dados.

Consideramos a necessidade imperiosa de orientar toda a equipe dos CAPS responsável pelo acolhimento para que possa seguir, em seu primeiro contato com o (a) usuário (a), os instrumentos de coleta fidedignamente, na busca de identificar, pela via dos registros de encaminhamento, medicações ou na fala do indivíduo, a existência de DCNTs, sustentando um acompanhamento das questões clínicas pré disponentes e de tantas outras passíveis de surgimento.

Nessa perspectiva, os profissionais de saúde devem estabelecer um processo terapêutico interdisciplinar e desenvolver habilidades e competências que atendam à pluralidade do ser humano. Entretanto, verifica-se que na prática profissional desses serviços há fragmentação do cuidado, reproduzindo o modelo biomédico especializado e centrado na doença mental.

Acreditamos na necessidade de realização de novos estudos que tragam dados sobre as DCNTs e sua relação com usuários dos serviços de saúde mental, a fim de difundirmos o assunto e levantarmos mais bibliografias acerca do apresentado, visando um cuidado de excelência, de forma integral e voltado à plenitude de cada usuário. Possibilitando novas ações, melhorando o acompanhamento e colocando em prática estratégias fundamentais no processo de cuidar.

Deste modo, a rede de atenção em saúde mental precisa desenvolver uma maior capacidade de percepção da saúde geral do usuário em saúde mental, no território e para as clínicas da família, referenciando-o, quando necessário, qualificando assim o fluxo entre os serviços de saúde. Na tentativa de responder, desenvolver ou até mesmo revisar os métodos de acesso e acompanhamento dos sujeitos, no intuito de atender suas necessidades de escuta onde se apresenta uma estruturação subjetiva que eventualmente acarreta uma geografia corporal diferenciada, uma vez que, em que pesem as diferenças inerentes aos usuários, a totalidade dos cuidados se contrapõe à perspectiva de tomá-lo em fragmentos, mas com necessidades justas e amplas. Tal articulação nos parece seguir, com maior proximidade, em consonância com os princípios e diretrizes propostos pelo Sistema Único de Saúde (SUS). 


\section{Referências}

Andreis, M., Cury, L., Johns, P., Albiero, M., Hassan, B., Hallal, A. L. C., Monteiro, A., \& Waetge, J. (2019). Doenças Crônicas Não Transmissíveis no Brasil. ACT Promoção da Saúde. NCD Alliance. https://ncdalliance.org/sites/default/files/resource_files/Civil\%20So ciety\%20Status\%20Report\%20ACT.pdf

Branquinho, J. S., Gomes, F. A., Silva, R. P., Leite, M. M. A., Candido, M. C. F. S., Lima, L. A., \& Bispo, I. M. G. P. (2014). Doenças crônicas em pacientes com transtornos mentais. Revista Eletrônica Gestão \& Saúde https://dialnet.unirioja.es/servlet/articulo?codigo=5558854

Brasil. (2005). A vigilância, o controle e a prevenção das doenças crônicas não-transmissíveis : DCNT no contexto do Sistema Único de Saúde brasileiro / Brasil. Ministério da Saúde. Organização Pan-Americana da Saúde. https://bvsms.saude.gov.br/bvs/publicacoes/DCNT.pdf

Brasil. (2013). Diretrizes para o cuidado das pessoas com doenças crônicas nas redes de atenção à saúde e nas linhas de cuidado prioritárias. Ministério da Saúde. Secretaria de Atenção à Saúde. Departamento de Atenção Básica. https://bvsms.saude.gov.br/bvs/publicacoes/diretrizes\%20_cuidad o_pessoas\%20_doencas_cronicas.pdf

Campos, R. O. (2001). Clínica: a palavra negada - sobre as práticas clínicas nos serviços substitutivos de Saúde Mental. Saúde em Debate. 25, 98-111, maio/ago. https://www.fcm.unicamp.br/fcm/sites/default/files/paganex/rosana2001clinicaapalavranegada.pdf

Chiavagatti, F.G., Kantorski, L. P., Willrich, J. Q., Cortes, J. M., Jardim, V. M. R., \& Rodrigues, C. G. S. S. (2012). Articulação entre Centros de Atenção Psicossocial e Serviços de Atenção Básica de Saúde. Acta Paul Enferm. https://www.scielo.br/pdf/ape/v25n1/v25n1a03.pdf

França, J. O. N., Ferreira, A. A., Lopez, T. A., Freitas, C. C. O., França, N. E. S., Cardoso, S. V., \& Brasil, V. B. P. (2021) Prevalência de comorbidades clínicas em portadores de transtornos mentais acompanhados no Centro de Atenção Psicossocial. Brazilian Journal of Health Review, 4, 1325-1342. 10.34119/bjhrv4n1114 https://www.brazilianjournals.com/index.php/BJHR/article/view/23221/18661

Hirdes, A. (2013). Apoio matricial em saúde mental: tecendo novas formas de relação e intervenção. Tese de Doutorado, PUC Rio Grande do Sul. 10.1590/180757622014.0710 https://www.scielo.br/pdf/icse/v19n53/1807-5762-icse-19-53-0405.pdf

Hirdes, A. (2015). A perspectiva dos profissionais da Atenção Primária à Saúde sobre o apoio matricial em saúde mental. Ciência \& Saúde Coletiva. 10.1590/1413-81232015202.11122014 https://www.scielo.br/pdf/csc/v20n2/1413-8123-csc-20-02-0371.pdf

https://www.teses.usp.br/teses/disponiveis/7/7141/tde-11092013-151204/en.php

Malta, D. C., Bernal, R. T. I., Lima, M. G., Araújo, S. S. C., Silva, M. M. A., Freitas, M. I. F., \& Barros, M. B. A. (2017) Doenças crônicas não transmissíveis e a utilização de serviços de saúde: análise da Pesquisa Nacional de Saúde no Brasil. Rev Saude Publica. https://doi.org/10.1590/S1518-8787.2017051000090 https://www.scielo.br/pdf/rsp/v51s1/pt_0034-8910-rsp-S1518-87872017051000090.pdf

Malta, D. C., Bernal, R. T. I., Neto, E. V., Curci, k. A., Pasinato, M. T. M., Lisboa, R. M., Cachapuz, R. F., Coelho, K. S. C., Santos, F. P., \& Freitas, M. I. F. (2020). Doenças Crônicas Não Transmissíveis e fatores de risco e proteção em adultos com ou sem plano de saúde. Ciência \& Saúde Coletiva, 25(8):2973-2983. 10.1590/1413-81232020258.32762018 https://www.scielo.br/pdf/csc/v25n8/1413-8123-csc-25-08-2973.pdf

Malta, D. C., Moura, L., \& Bernal, R. T. I. (2015). Diferenciais dos fatores de risco de Doenças Crônicas não Transmissíveis na perspectiva de raça/cor. Ciência \& Saúde Coletiva. 10.1590/1413-81232015203.16182014 https://www.scielo.br/pdf/csc/v20n3/pt_1413-8123-csc-20-03-00713.pdf

Malta, D. C., Stopa, S. R., Santos, M. A. S., Andrade, S. S. C. A., Oliveira, M. M., Prado, R. R., \& Silva, M. M. A. (2017). Fatores de risco e proteção de doenças e agravos não transmissíveis em adolescentes segundo raça/cor: Pesquisa Nacional de Saúde do Escolar. Revista Brasileira de Epidemiologia. 10.1590/19805497201700020006 https://www.scielo.br/pdf/rbepid/v20n2/1980-5497-rbepid-20-02-00247.pdf

Nasi, C., \& Schneider, J. F. (2011). O Centro de atenção psicossocial no cotidiano dos seus usuários. Rev Esc Enferm USP. 45(5):1157-63 https://www.scielo.br/pdf/reeusp/v45n5/v45n5a18.pdf

Nasi, C., Cardoso, A. S. F., Schneider, J. F., Olschowsky, A., \& Wetzel, C. (2009). Conceito de integralidade na atenção em saúde mental no contexto da reforma psiquiátrica. Rev. Min. Enferm. 13(1): 139-146, jan./mar. https://cdn.publisher.gn1.link/reme.org.br/pdf/v13n1a20.pdf

Pereira, A. S., Shitsuka, D. M., Parreira, F. J., \& Shitsuka, R. (2018). Metodologia da pesquisa científica. Santa Maria, RS. $1^{\text {a }}$ edição. UAB/NTE/UFSM. Universidade Federalde Santa Maria. https://repositorio.ufsm.br/bitstream/handle/1/15824/Lic_Computacao_Metodologia-Pesquisa-Cientifica.pdf?sequence=1

Pimenta, F. B., Pinho, L., Silveira, M. F., \& Botelho, A. C. C. (2015). Fatores associados a doenças crônicas em idosos atendidos pela Estratégia de Saúde da Família. Ciência \& Saúde Coletiva. 20(8):2489-2498. 10.1590/1413-81232015208.11742014 https://www.scielo.br/pdf/csc/v20n8/1413-8123-csc-20-082489.pdf

Próchno, C. C. S. C., \& Bessa, W. L. C. (2016). Os Efeitos da Contemporaneidade nos Centros de Atenção Psicossocial (CAPS). Rev Subjetividades, Fortaleza. 10.5020/23590777.16.3.21-33 https://periodicos.unifor.br/rmes/article/view/5208/pdf

Santos, A. M. C. C. (2009). Articular saúde mental e relações de gênero: dar voz aos sujeitos silenciados. Ciência \& Saúde Coletiva. 14(4):1177-1182, 2009 https://www.scielo.br/pdf/csc/v14n4/a18v14n4.pdf

Santos, J. C. (2013). O estigma da doença mental: compreensão e ações dos trabalhadores dos CAPS. Dissertação (Mestrado em Cuidado em Saúde) - Escola de Enfermagem, University of São Paulo, São Paulo. 10.11606/D.7.2013.tde-11092013-151204 https://teses.usp.br/teses/disponiveis///7/7141/tde-11092013151204/pt-br.php

Santos, V. C. F., Kalsing, A., Ruiz, E. N. F., Roese, A., \& Gerhardt, T. E. (2013). Perfil das internações por doenças crônicas não-transmissíveis sensíveis à atenção primária em idosos da metade sul do RS. Rev Gaúcha Enferm. 34(3):124-131. https://www.scielo.br/pdf/rgenf/v34n3/a16v34n3.pdf

Secretaria de Estado de Saúde do Espiríto Santo. (2018). Diretrizes clínicas em saúde mental. Vitória, ES. https://saude.es.gov.br/Media/sesa/Protocolo/Diretrizes\%20Clinicas\%20em\%20saude\%20mental.pdf 
Research, Society and Development, v. 10, n. 7, e34110716717, 2021

(CC BY 4.0) | ISSN 2525-3409 | DOI: http://dx.doi.org/10.33448/rsd-v10i7.16717

Silva, N. G., Barros, S., Azevedo, F. C., Batista, L. E., \& Policarpo, V. C. (2017). O quesito raça/cor nos estudos de caracterização de usuários de Centro de Atenção Psicossocial. Saúde Soc. 26, 100-114. 10.1590/S0104-12902017164968 https://www.scielo.br/pdf/sausoc/v26n1/1984-0470-sausoc-26-01-00100.pdf

Tavares, N. U. L., Costa, K. S., Mengue, S. S., Vieira, M. L. F. P., Malta, D. C., \& Silva Júnior, J. B. (2015). Uso de medicamentos para tratamento de doenças crônicas não transmissíveis no Brasil: resultados da Pesquisa Nacional de Saúde, 2013. Epidemiol. Serv. Saúde, Brasília, 24(2): 315-323, 10.5123/S167949742015000200014 https://www.scielo.br/pdf/ress/v24n2/2237-9622-ress-24-02-00315.pdf 